\title{
Chapter
}

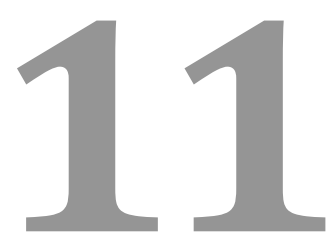

\section{TEMPOROMANDIBULAR DISORDERS AND}

BIO-IPNS: IN VITRO APPROACH TO FIND MOLECULAR SOLUTION TO BIOLOGICAL PROBLEM

\section{Tamara Perchyonok ${ }^{1,2^{*}}$, Sias Grobler ${ }^{3}$, Nicollaas Basson ${ }^{3}$,} Desigar Moodley ${ }^{3}$, and Shengmiao Zhang ${ }^{4}$

${ }^{1}$ Health Innovations Research Institute, RMIT University, Melbourne, Australia, 3001

2 VTPCHEM PTY LTD, Research and Development, Southport, Australia 4215

${ }^{3}$ Oral and Dental Research Institute, Faculty of Dentistry, University of the Western Cape, Private Bag X1, Tygerberg 7505, Cape Town, South Africa ${ }^{4}$ School of Material Science and Engineering, East China University of Science and Technology, 130 Meilong Road, Shanghai, 200 237, China 


\section{Contents}

11.1. INTRODUCTION

11.1.1. Introduction to temporomandibular disorders: anatomy and physiology summary 265

11.1.1.1. Temporomandibular disorders: general introduction

11.1.1.2. Myogenic disorders 267

11.1.1.3. Articular disorders

11.2. FREE RADICALS AND TEMPOROMANDIBULAR JOINT IN ACTION 268

11.3. AETIOLOGY OF TEMPOROMANDIBULAR JOINT IN A NUTSHEL ...................................269

11.4. PATHOGENESIS OF TEMPOROMANDIBULAR JOINT UP TO DATE.................................270

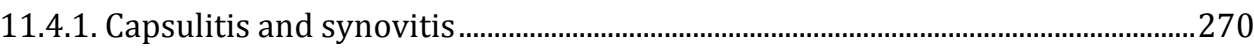

11.4.2. The artritides

11.5. POTENTIALS SOLUTIONS TO THE TEMPOROMANDIBULAR JOINT PROBLEM THROUGH BIOMATERIALS.

11.5.1. Temporomandibular joint and regeneration scaffolds .......................................22

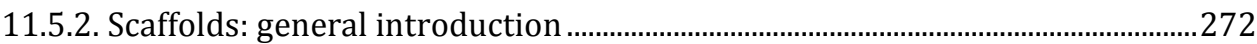

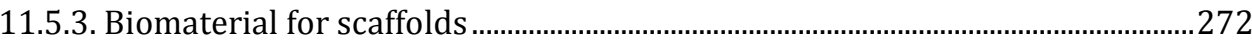

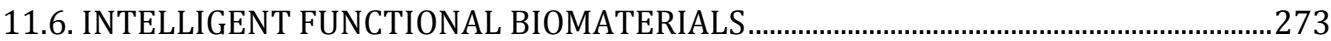

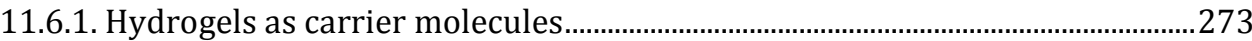

11.6.2. Interpenetrating polymeric network hydrogels as a topical drugdelivery system in the oral environment....................................................................273

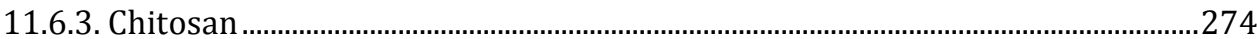

11.6.4. Temporomandibular joint bioengineering: general introduction......................2 275

11.6.5. Temporomandibular joint disc bioengineering up to date summary..............275

11.6.6. Chitosan/gelatin/hydroxyapatite scaffolds as potential biomaterials for hard tissue regeneration: in vitro approach ...........................................................276

11.6.7. Chitosan/hydroxyapatite for bone/hard tissue engineering .............................277

11.6.8. Composite materials for bone tissue engineering .................................................278

11.6.9. Carbon nano tubes for bone tissue engineering ...................................................279

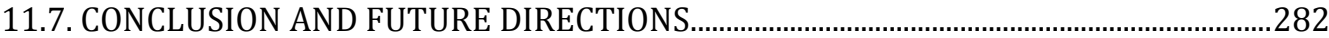

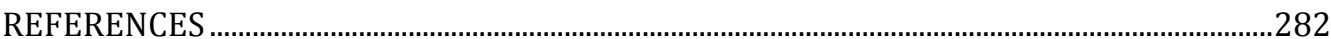




\subsection{INTRODUCTION}

In the last few decades, tissue engineering has emerged as a promising multidisciplinary approach for the repair and regeneration of damaged bone tissue.

The molecular events that underline the degenerative temporomandibular joint (TMJ) diseases are poorly understood. Mechanical stresses are generated during functional or para-functional movements of the jaw, adaptive mechanism of the TMJ may be exceeded by free radical accumulation leading to a dysfunctional state (i.e. disease state). Biologically relevant free radicals are very reactive and unstable molecular entities that have an unpaired electron and they can produce participate in the propagation chain reaction to form a new radical. Although oxygen free radicals participate in many physiological processes, they can be harmful to tissue when either their action or their generation have been left uncontrolled. The most common source of free radicals in biological systems is oxygen. The elevation of reactive oxygen species (ROS) lead to oxidative stress that causes molecular damage to the vital structures and functions.

This chapter addresses the principal intrinsic and extrinsic factors that impede integration and describe how manipulation of these factors using a host of strategies can positively influence cartilage integration based on the designer biomaterial scaffolds and combinations designed by others and us in vitro development.

\subsubsection{Introduction to temporomandibular disorders: anatomy and physiology summary}

Signs and symptoms of temporomandibular disorders (TMDs) may include pain, impaired jaw function, malocclusion, deviation or deflection, limited range of motion, joint noise, and locking. Headache, tinnitus, visual changes, and other neurologic complaints may also accompany TMDs. Because of many etiologic factors, the diagnosis and treatment of patients with TMDs is complex. TMDs can be subdivided into muscular and articular categories. Differentiation between the two is sometimes difficult because muscle disorders may mimic articular disorders, and they may coexist. Myogenic disorders include myalgia (myofascial pain (MFP), fibromyalgia), myospasm, splinting, and fibrosis / contracture. Articular disorders include synovitis / capsulitis, joint effusion, trauma / fracture, internal derangement, arthritis, and neoplasm.

TMJ is a compound articulation formed from the articular surfaces of the temporal bone and the mandibular condyle. Both surfaces are covered by dense articular fibrocartilage. Each condyle articulates with a large surface 
area of temporal bone consisting of the articular fossa, articular eminence, and preglenoid plane. The TMJ functions uniquely in that the condyle both rotates within the fossa and translates anteriorly along the articular eminence. Because of the condyle's ability to translate, the mandible can have a much higher maximal incisal opening than would be possible with rotation alone. The joint is thus referred to as "gynglimodiarthrodial": a combination of the terms gynglymoid (rotation) and arthroidial (translation) [1].

A cartilaginous disc resides between the articular surfaces of the temporal bone and mandibular condyle. Although other articular cartilages are composed of hyaline cartilage, this disc is composed of fibrocartilage; thus, the disc contains a much higher percentage of collagen, increasing its stiffness and durability. The disc does not have any direct vascularization or innervation; however, the posterior attachment of the disc (also known as retrodiscal tissue) is both highly vascularized and highly innervated and, therefore, pertinent to the discussion of joint pain. The superior lamina of the retrodiscal tissue limits extreme translation, whereas the inferior lamina limits extreme rotation. The lateral pterygoid muscle controls the opening of the mandible. The superior segment of this muscle attaches to the anterior portion of the disc, and the inferior segment attaches inferior to the condyle. As both segments contract the condyle translates anteriorly along the articular eminence, and the disc remains interposed between the condyle and the temporal bone at all points of translation.

The joint is stabilized by three ligaments: collateral (discal), capsular, and temporomandibular. These attach to the disc at the medial and lateral poles of the mandibular condyle, as well as to the temporal fossa. These ligaments limit extreme condylar movement. The capsular ligament surrounds the joint space and disc and acts to contain the synovial fluid within the joint space.

The capsule is lined by a synovial membrane. Synovial tissue covers all intra-articular surfaces except for the pressure-bearing fibrocartilage (i.e. disc, condyle, eminence). The synovial tissue is highly innervated and vascularized and has regulatory, phagocytic, and secretory functions. The synovial fluid has metabolic and nutritional functions and is essential to joint surface lubrication [2].

The masseter, medial pterygoid, lateral pterygoid, and temporalis muscles are the muscles of mastication. The masseter, medial pterygoid, and temporalis are primarily responsible for mandibular closure and bite force, whereas the lateral pterygoid and infrahyoid muscles are responsible for mandibular opening. Mandibular movement is also influenced by the digastric, geniohyoid, mylohyoid, stylohyoid, sternohyoid, omohyoid, sternothyroid, and thyrohyoid muscles, which as a group coordinate complex mandibular movements including opening, protrusion, retrusion, lateral excursion, and closure.

At rest, the condyle is seated passively in the temporal fossa with the fibrocartilage disc interposed at the most superior and anterior position of the 
condyle. Mandibular opening commences with contraction of the lateral pterygoid and infrahyoid muscles, which rotates the condyle. Mandibular opening proceeds with lateral pterygoid contraction pulling the condyle forward along the articular eminence (translation). The superior segment of the lateral pterygoid muscle coordinates the translation of the disc with the condyle. During jaw closing the ligaments and retrodiscal lamina pull the condyle and disc back into resting position.

The TMJ receives its vascular supply from the superficial temporal, maxillary, and masseteric arteries. Innervation of the joint is provided mainly by the auriculotemporal nerve and, to a lesser extent, the masseteric and posterior deep temporal nerves. The production of synovial fluid is also under a certain amount of neuronal control.

\subsubsection{Temporomandibular disorders: general introduction}

The term "TMJ pain" varies greatly in meaning among clinicians, patients, and the general population. Historically, symptom-based classification of the disorder has been problematic. As stated by Laskin $[3,4]$, the difficulty began with the introduction of a "TMJ syndrome." Then clinicians erroneously grouped a "variety of etiologically unrelated conditions into one diagnostic category based on the fact that they produced similar signs and symptoms," and this led to "one diagnosis equals one treatment." Only later was it recognized that many of these patients suffered from muscle-related conditions. The terms MFP and myofascial pain and dysfunction (MPD) evolved [5], and "TMJ disorders" became "TMDs." were differentiation of the TMDs into articular (joint) and nonarticular (myogenic) disorders can be made and will be further discussed.

\subsubsection{Myogenic disorders}

Traditionally, it was thought that structural abnormalities (i.e. dental malocclusion, condylar malposition) led to muscular dysfunction and pain $[6,7]$. Muscles were thought to be under an increased burden in the presence of these skeletal and / or dental misalignments. As such, a "vicious cycle" model was proposed: Structural $\rightarrow$ abnormality $\rightarrow$ muscle hyperactivity $\leftrightarrow$ pain $\leftrightarrow$ mandibular dysfunction where pain and muscle hyperactivity potentiate each other and emotional stress is thought to have an additive effect [6,7]. Over time, there has been a lack of scientific evidence to support this theory.

MFP of the masticatory muscles is more frequently induced by stress-related parafunctional habits (i.e. clenching and grinding) and rarely by mechanical causes such as occlusal prematurities or high dental restorations. MFP and MPD, although considered to be muscular disorders, are thought to possibly play a causative role in degenerative disease of the TMJ. 


\subsubsection{Articular disorders}

The etiology of articular disorders may be degenerative, traumatic, infectious, immunologic, metabolic, neoplastic, congenital, or developmental.

Articular disc displacement (internal derangement) Anterior disc displacement (ADD) is the most frequently encountered articular disorder. Disc displacement (also known as internal derangement) is defined as "a disturbance in the normal anatomic relationship between the disc and condyle that interferes with smooth movement of the joint and causes momentary catching, clicking, pop-ping, or locking" [8-11].

The ability of the joint to adapt to biomechanical stress and disc derangement has been a subject of debate. In his classification system, Wilkes [12] promotes the theory that internal derangement logically progresses to degenerative joint disease (DJD). Historically, surgical and nonsurgical approaches have been used to reposition the displaced disc, with the goal of arresting this progression [13]. In an opposing view, Milam [9] states that "the adaptive capacity of the TMJ is not infinite ...some individuals are... capable of mounting an adaptive response to an articular disc displacement; other individuals may not adapt to these structural derangements, and a progressive DJD may result." Factors considered to compromise the adaptive response include age, sex, stress, and illness $[9,14]$. He concludes that disc derangement may exist variably as cause or effect, but does not always progress to disease.

\subsection{FREE RADICALS AND TEMPOROMANDIBULAR JOINT IN ACTION}

Mechanical stresses are generated during functional or para-functional movements of the jaw, adaptive mechanism of the TMJ may be exceeded by free radical accumulation leading to a dysfunctional state (i.e. disease state). Biologically relevant free radicals are very reactive and unstable molecular entities that have an unpaired electron and they can produce participate in the propagation chain reaction to form a new radical. Although oxygen free radicals participate in many physiological processes, they can be harmful to tissue when either their action or their generation have been left uncontrolled. The most common source of free radicals in biological systems is oxygen. The elevation of ROS lead to oxidative stress that causes molecular damage to the vital structures and functions. ROS are generated on a regular basis in biological pathways as a product or as a signal transducer.

However, excessive production or ineffective scavenging of ROS can cause over accumulation, which can injure or kill cells. All basic molecules in living organisms can be attacked by ROS, e.g., lipids, carbohydrates, proteins and nucleic acids [15-17]. 
The unsaturated fatty acids of cell membrane lipid are susceptible to peroxidative reaction. Lipid peroxidation of cell membranes has been implicated in the wide range of tissue injuries and diseases. Accumulation of lipid hydroperoxides in a membrane disrupts its function and causes it to collapse and have range of cytotoxic radicals. The most series of which are aldehydes. They may also react with transition metals like iron or copper to form stable aldehyde such as malodialdehyde for example, which will damage the cell membrane. Because hemoglobin constitutes the largest iron store in the body, it is speculated to be a potential source of redox activity iron, which can catalyze the formation of free radicals that might be damaging to the joint [18].

Antioxidant defense mechanisms involve both enzymatic and non-enzymatic strategies. Common antioxidants include the vitamins A, C and E, glutathione, and the enzymes superoxide dismutase, catalyse, glutathione peroxidase and glutathione reductase. They work in synergy with each other and against different types of free radicals. Antioxidant enzymes in living organisms have evolved as very sophisticated and effective scavengers of ROS. Superoxide dismutase (SOD) is an essential antioxidant enzyme protecting many cellular components by converting two superoxide anions into a molecule of hydrogen peroxide and one molecule of oxygen. SODs are found in many organisms including all oxygen-consuming organisms [19].

\subsection{AETIOLOGY OF TEMPOROMANDIBULAR JOINT IN A NUTSHEL}

Most of the scientists explain that osteoarthritis (OA) as an inflammatory process, TMJ disorder beingthe most frequent one, is characterised by proliferative changes in the synovial and primary degeneration of the cartilage and surrounding tissues [20,21]. It is found that $28 \%$ of the adult population have symptoms and clinical signs of TMD [22,23]. The aetiological factors of TMJ disorders as follows: systemic diseases (rheumatoid arthritis (RA), psoriasis, pseudogout, ankylosing spondylitis, etc.), secondary inflammatory component from the neighbouring regions (otitis, maxillary sinusitis, tonsillitis), trauma (chronical), prevalence of dental arch defects e.g. missing of molar teeth [24], malocclusion, endocrinological disturbances, odontogenic infections (impacted third molars). Presence of specific bacterial organisms such as Staphylococcus aureus, Streptococcus mitis, Mycoplasma fermentas, Actinobacillus actinomycetemcomitans in the synovial fluid have been found [25]. Serum antibodies against Chlamydia species in patients with monoarthritis of the TMJ have also been reported [26].

Although patients without internal derangement may develop OA [27], a complex two-way relationship exists. Controversy continues as to whether disc 
derangement is a cause or a result of DJD; however, scientific evidence strongly supports the latter conclusion [27-31].

\subsection{PATHOGENESIS OF TEMPOROMANDIBULAR JOINT UP TO DATE}

Inflammation mainly affects the posterior disc attachment [20,27]. Several inflammatory mediators play an important role in the pathogenesis of TMJ disorders like tumor necrosis factor alpha (TNF- $\alpha$ ), interleukin-1beta (IL-1 $\beta$ ), prostaglandin E2 (PGE2), leukotriene B4 (LkB4), matrix metalloproteinases (MMPs), serotonin or 5-hydroxytryptamine (5-HT) [22,28]. MMPs are the early marker or detector to determine TMJ arthritis [27]. Serotonin is the mediator of pain and inflammation is produced in enterocromaffin cells of the gastrointestinal mucosa and absorbed by platelets. It is also produced in the synovial membrane and in the synovial fluid which causes TMJ pain in cases of systemic inflammatory joint diseases [28-31]. Inflammation results in tissue response as: vasodilatation, extra vasation, release of mediators, activation of nociceptors, release of neuro peptides as substance P (SP), neuropeptide Y (NPY), which stimulate release of inflammatory mediators like histamine and serotonin and hyperalgesia.

\subsubsection{Capsulitis and synovitis}

Inflammation of the capsular ligament may manifest with swelling and continuous pain localized to the joint. Movements that stretch the capsular ligament cause pain with resultant limitation of such movement. Significant inflammation may increase joint fluid volume. When this occurs, one may see an ipsilateral posterior open bite (lack of contact between maxillary and mandibular teeth) secondary to inferior displacement of the condyle [7]. Similarly, inflammation due to trauma or abnormal function may affect the retrodiscal tissue. Edema in this area may cause anterior displacement of the condyle and an acute malocclusion with painful limitation of mandibular movements.

The highly innervated and vascularized synovial membrane digests debris and pain mediators released from cartilage degradation. When this ability is overwhelmed, inflammation (acute synovitis) results. Inflammation of the synovial membrane is an early sign of DJD [32]. Inflammatory and pain mediators have been identified in TMJ synovial fluid [33,34]. Chemical breakdown of degenerative byproducts is thought to stimulate the production of inflammatory and pain mediators (PGE2 and LkB4, among others) through the arachidonic acid cascade. PGE2 is a powerful vasodilator and LkB4 attracts inflammatory cells. Their presence creates acute synovitis pain and stimulates further damage from cytokines and proteases. For this reason arthrocentesis and arthroscopy for joint lavage and lysis of adhesions are believed to have a 
therapeutic effect [35-37]. These procedures remove particulate debris and pain mediators, aiding reduction of joint inflammation and pain. Results are similar with and without disc repositioning [35]. Lysis of adhesions may improve range of motion. Steroid injections are also used to reduce synovial inflammation and pain. Recent investigations have looked at intra-articular morphine for sustained pain relief in patients [37]. Research is now focusing on the role of biochemical mediators in the development and progression of TMJ pain and dysfunction $[19,33]$ and the identification of biochemical "markers" of TMJ disease [14].

\subsubsection{The artritides}

Arthritis of the TMJ has many etiologies: frequently OA and RA and less often infectious, metabolic (gout), or immunologic (ankylosing spondylitis, lupus). DJD, also known as OA, has a multifactorial pathogenesis including biomechanical, biochemical, inflammatory, and immunologic insults. Excessive and repetitive mechanical stress has been implicated [19]. Inflammatory mediators and waste products may play a role in DJD [34-49]. Inflammatory states cause changes in the viscosity of synovial fluid, which changes its ability to nourish the articular cartilage, thus changing cartilage metabolism.

\subsection{POTENTIALS SOLUTIONS TO THE TEMPOROMANDIBULAR JOINT PROBLEM THROUGH BIOMATERIALS}

\subsubsection{Temporomandibular joint and regeneration scaffolds}

The craniofacial structure consists of bone, cartilage, soft tissue, nerves, and blood vessels. Acquired defects after cancer surgeries, trauma as well as congenital or developmental deformities require a reconstructive procedure as the bones of the craniofacial region support the rest of the elements. The procedures used today for temporomandibular reconstruction are mostly autologous, allogenic, or alloplastic, with variable clinical outcomes and morbidities [50]. Distraction histogenesis has emerged as a possible alternative to regenerate ramus condyle unit [51].

Regeneration using osteoconductive scaffolds, osteoinductive growth factors, committed progenitor cells and stem cells is being investigated by researchers and surgeons alike [52]. Osteoconduction and osteoinduction are very important features for bone tissue scaffolds. Osteoinduction implies the process of the conversion of non-osseous cells into bone forming cells, whereas osteoconduction is the process by which implanted scaffold supports the bone growth [53]. 
The ideal bone construct for repair should be able to replicate the lost structure, restore function, be harmless, reliable and biodegradable i.e. should degrade during the process of tissue regeneration and replaced with fully functional tissue.

\subsubsection{Scaffolds: general introduction}

A crucial requirement for joint repair is that the scaffolding should be attached to the cartilage lesions and should integrate with the tissue. Not only this but also the attachment must balance temporary mechanical function with mass transport to aid biological delivery and tissue engineering. In addition to being patient specific, the scaffolds should facilitate cell attachment and regulate cell differentiation. Also they must be biodegradable, with nontoxic by-products, and exhibit favourable resorption kinetics to maintain initial stability.

The materials can be divided into natural or synthetic, based on the sources. Natural scaffolds may be subdivided into [54] protein based matrices such as collagen and fibrin [55], mineral-based matrices such as autogenous, allogenic and xenogenic bone grafts, and [56] carbohydrate-based matrices such as alginate, agarose, chitosan (CTS), and hyaluronan. Synthetic materials have been used extensively both in vitro and in vivo. They include poly(lactic acid) (PLA), poly(glycolic acid) (PGA), polycaprolactone (PCL) and their derivatives, for example poly(lactic-co-glycolic acid) (PLGA). The synthetic materials have been popular because of their easy moulding characteristics, relatively easy production, and the ability to control dissolution and degradation. However, their major weakness is biocompatibility. They are degraded by a hydrolytic reaction, thereby high concentrations of acidic by-products and particulates can be released, causing inflammation, giant cell reaction and chondrocyte death owing to a reduction in $\mathrm{pH} 50$.

\subsubsection{Biomaterial for scaffolds}

The major materials used in craniofacial tissue engineering are natural and synthetic polymers, ceramics, composite materials, and electrospun nanofibers [57]. Biomaterial to be used as a scaffold must possess sufficient mechanical strength, large pore volumes and pore interconnectivity to allow continuous tissue in growth, and transport properties to allow the influx of nutrients and elimination of waste products [58]. Randomly positioned pores contribute to better cell seeding and better cell aggregation in the designed scaffolds [59]. Natural scaffolds like collagen type I, CTS, calcium alginate, hyaluronic acid, and composites have been shown to be osteoconductive, but with problems like lack of mechanical strength when implanted, risk of infection, immunogenicity, and rapid degradation rate [60-65].

Bone contains $85 \%$ calcium phosphate, hence ceramics such as hydroxyapatite (HAp), tricalcium phosphate (TCP), and composites such as biphasic calcium phosphate (BCP), have been widely investigated for bone scaffolds. The HA 
ceramics are well suited as biomaterials because of their biocompatibility, not eliciting an inflammatory response, lack of immune reaction, and easy radiographic assessment. TCP demonstrates a too fast degradation rate in vivo, whereas HA degrades too slowly, is not resorbed, and resides in the defect for several years after callus formation. BCP has more favorable degradation rates compared with TCP and HA. However, the problem with use of ceramics is their brittleness which makes them mechanically inadequate for load bearing [65-67].

Polymers include poly(ethylene glycol) (PEG), PGA, poly-L-lactic acid and poly(D-lactic acid) (PLA), PLGA, PCL, poly-urethanes, and composites. Polymers are flexible and biode-gradable through their hydrolysis or by means of cellular or enzymatic pathways when implanted. Polymers have low mechanical strength and hence are often combined with high-modulus micro or nanoscale ceramic constituents like HA.

\subsection{INTELLIGENT FUNCTIONAL BIOMATERIALS}

\subsubsection{Hydrogels as carrier molecules}

The idea of developing a drug that selectively destroys diseased cells without damaging healthy cells was proposed by the Nobel Prize winner Paul Ehrlich, almost a century ago. He called this hypothetical drug the "magic bullet" [68]. Today, several decades later, many scientists have focused their attention on the development of ideal drugs that specifically target the site of action. Such a targeted drug-delivery system needs three components: a therapeutic agent, a targeting moiety, and a carrier system. The choice of the carrier molecule is of high importance, since it can significantly affect the pharmacokinetics and pharmacodynamics of the drugs [69,70]. Hydrogels are natural polymers that have been widely used as carrier systems into which; the drug can be incorporated by passive absorption or chemical conjugation. Hydrogels are biocompatible hydrophilic three-dimensional matrices that can act as drug carriers and protectors, especially for peptide and protein-based drugs. Because of their hydrophilic characteristics, hydrogels have improved bioadhesive properties, which make them suitable carrier materials for sustained topical drug-delivery systems for targeted treatment of some of the oral diseases $[71,72]$.

\subsubsection{Interpenetrating polymeric network hydrogels as a topical drug-delivery system in the oral environment}

Multicomponent drug-delivery systems have found several potential diagnostic and therapeutic applications. Among these, the interpenetrating polymeric network (IPN) has emerged as one of the most useful functional biomaterial. IPNs are entanglement of polymers with at least one network 
synthesized and / or cross-linked in the presence of the other. They are ideally held together only by a permanent topological interactions [73], presenting a three-dimensional network structure that provides free volume space for an easy encapsulation of drugs.

Many researchers suggest that specifics IPN characteristics, such as, non-separable network, adhesive property, high-tensile strength and biocompatibility enabled the enhancement of the implementation of these hydrogels. Furthermore, IPNs are distinguishable from blends, block copolymers, and graft copolymers for two important reasons; firstly, an IPN swells but does not dissolve in solvents, and secondly, the creep and flow is suppressed. Because of its distinguishable features, these biocompatible, nontoxic and biodegradable polymers are acquiring a unique place in various biomedical applications, such as, cartilage scaffolds, biological tissue graft, tissue engineering, wound dressing and for drug delivery [74-76].

IPNs can be prepared for different applications and by using various matrices such as poly-urethane, polybutadiene, methacrylic acid, L-lysine, glutamic acid, poly(vinyl alcohol), carboxymethyl cellulose, poly(acrylic acid), gelatin, poly(vinylpyrrolidone), alginate, dextran, xanthane, guar-gum, CTS, PEG, HAp, etc. [77-83].

The newly designed hydrogel system, bioactive-functionalized interpenetrating network (BIOF-IPN) hydrogel, presented in this chapter represents a novel interplay between bio-molecular scaffold designs with functionality. The system optimizes the use of the host : guest science playing an important role as a "build-in" free radical defense mechanism, and acting as a "proof of concept" for the functional multi-dimensional restorative repair materials. This chapter reports the preliminary investigations of the BIOF-IPN utilizing a combination of materials such as CTS in the restorative and regenerative dental field specifically aimed at TMJ in vitro evaluations [84-93].

\subsubsection{Chitosan}

CTS is a natural, biodegradable, non-toxic, mucoadhesive and biocompatible polymer that has garnered immense attention in the pharmaceutical field, inclusive as a vehicle for oral drug-delivery. The suitability of CTS based IPN hydrogels stems from their capability of imbibing large amount of body fluid without solubilisation, great potential for encapsulation of a large amount of drugs, and adaptability to be combined with specific responsive polymer(s).

Furthermore, it presents good biocompatibility, low toxicity and unique biological and physical-chemical characteristics [94]. The primary reactive functional groups (hydroxyl and amine groups) located on the backbone of CTS allow for chemical modification to control its mechanical and solubility properties. When a hydrophobic moiety is conjugated to a CTS molecule, a chemically cross-linked three-dimensional nanoparticle is formed that can physically (entanglement) or chemically, encapsulate a number of drugs. When 
applied to a specific site of action, the immobilized active agents or biomolecules are released in a well-defined and precise manner [95].

CTS is metabolized by certain human enzymes, especially lysozyme, and is considered biodegradable [96]. It is also a non-immunogenic, non-carcinogenic and mucoadhesive polymer, which makes it a suitable candidate for biomedical applications, such as wound management, tissue engineering and drug delivery vehicle [97-101]. CTS as well shows permeation enhancer properties, and it can improve the paracellular route of absorption, which is important for the transport of hydrophilic compounds such as therapeutic peptides and antisense oligonucleotides across the membrane [102-104].

CTS has generated considerable interest as a bioadhesive material. The interactions are strong at acidic and slightly acidic $\mathrm{pH}$ levels in oral environment, at which the charge density of CTS is high, and an increase in the molecular weight of CTS results in a stronger adhesion [105]. CTS also displays additional biological properties viz., regenerative effect on connective gingival tissue, accelerates formation of osteoblast, is haemostatic, fungistatic, spermicidal, antitumor, anticholesteremic and immune adjuvant [106].

\subsubsection{Temporomandibular joint bioengineering: general introduction}

Ideal engineered constructs for mandibular condyle regeneration must have integrated bone and cartilage layers in a single osteochondral construct to meet the demands for anatomic, structural, and functional regeneration. The challenge in TMJ bioengineering is to promote matrix synthesis and tissue maturation of stem-cell-derived chondrogenic and osteogenic cells in biocompatible and bioactive scaffolds, which may be possible by incorporating an array of growth factors and/or transcription factors separately for chondrogenesis and osteogenesis. The mechanical properties of the tissue-engineered mandibular condyle must match with that of an anatomic condyle for in situ implantation into the human TMJ. Also, the tissue-engineered mandibular condyle must have a remodeling potential [107].

\subsubsection{Temporomandibular joint disc bioengineering up to date summary}

The earliest such study was performed in rabbit disc where cultured cells were used in collagen I meshes [108]. Later hyaline cartilage was engineered in the shape of a human TMJ disc [109]. Four years later, Girdler harvested hyaline cartilage cells along with chondroprogenitor cells and cultured them to form disc [110]. Recently, human and porcine disc cells have been cultured in 2 dimensions on expanded poly(tetrafluorethylene) monofilaments, PLA monofilaments, polyamide mono-filaments, and natural bone mineral blocks [111]. 
Recent studies have identified that a scaffold of non-woven PGA mesh in combination with cell seeding technologies, could provide an engineered disc [112]. Three growth factors: insulin-like growth factor-I, basic fibroblast growth factor and transforming growth factor- $\beta 1$ have been assessed in maintaining disc-like tissue in culture $[113,114]$.

In another study, a scaffold material composed of porcine-derived extracellular matrix, configured to mimic the shape and size of the TMJ, was implanted in a canine model of bilateral TMJ discectomy. The results showed the formation of site-appropriate, functional host tissue resembling native TMJ disk [115].

Poly(glycerol sebacate) (PGS), a biocompatible, biodegradable elastomer, was used as a porous scaffold material for the TMJ disc, where goat fibrochondrocytes were seeded at three seeding densities $(25,50,100$ million cells $\mathrm{mL}^{-1}$ scaffold), respectively, and cultured. The results showed that cell seeding density and culture time, both effect the biochemical and biomechanical properties of PGS scaffolds. The findings demonstrated PGS as a favorable scaffold material for TMJ disc engineering [116].

\subsubsection{Chitosan/gelatin/hydroxyapatite scaffolds as potential biomaterials for hard tissue regeneration: in vitro approach}

Bones are rigid organs that support and protect various organs of the body. Repair techniques for bones with defects or loss due to disease, trauma or tumor resections are the subject of intensive research [117]. Bone grafts - a well recognized and standard treatment method for reconstructive orthopedic surgery [118] - employ three types of bone or tissue substitution: allograft, autograft and xenograft. Allografts transplant bone or soft tissue from one individual to another in the same species. Allografts have many advantages, including osteoinduction and strong mechanical properties, but carry the risk of disease transmission from the donor, such as HIV, hepatitis or cancer. Autografts, or autologous bone transplants, transplant bone tissue from one site to another in the same individual. This graft offers excellent biocompatibility and does not stimulate host inflammatory response. However, the procedure may cause long lasting pain and discomfort for the patient. Furthermore, there is additional risk of wound infection at the surgery sites [119]. Xenografts remove cells or sections of tissue from one species and graft them on or into a different species. Bovine bone [120] and mollusk shell [121] are common materials used in xenografts. However, the bioactive properties of xenografts are weaker than allografts and autografts. To improve xenografts, a new treatment technique has been introduced for bone or tissue repair called bone tissue engineering - a procedure to regenerate damaged bone by implanting cells, proteins and scaffold to provide mechanical support for gap areas [122]. Bone substitute morphology has many forms, such as a compact and porous structure. As bone replacements, a compact structure provides good mechanical strength while a porous structure is suitable for cell 
attachment and blood supply [123]. Bone tissue engineering creates a biological material that provides the option for implantation and/or prosthesis. Bone tissue engineering has three main requirements: osteoconductive biomaterial scaffolds, osteogenic cells and osteoinductive molecules [124]. Materials widely used in bone tissue engineering include CTS, gelatin and HA. CTS is a polysaccharide that can be synthesized from crustacean shell and squid pen. CTS's structure is similar to glycosaminoglycans, the major component of the extracellular matrix of bone and cartilage $[125,126]$. Gelatin can be obtained by thermal denaturation and chemical degradation of collagen [127], and is known to benefit cell viability [128]. In the meantime, HA has a chemical composition similar to human mineral tissue and can be synthesized from many natural sources with calcium-based structures, such as bovine bone, mollusk shell and coral [129-131]. In combination, these three biomaterials offer potential synergies between physical properties and bioactivity for use as bone substitutes in bone grafts, benefiting a range of surgical applications.

\subsubsection{Chitosan/hydroxyapatite for bone/hard tissue engineering}

Research on biomaterials for bone implantation and replacement has expanded considerably over the last four decades. In recent years, significant progress has been made in organ transplantation, surgical reconstruction and the use of artificial prostheses to treat the loss or failure of an organ or bone tissue. The establishment of a load bearing biomaterial must be incorporated with natural bone. The implanted biomaterial should possess the following criteria: biocompatibility, osteoconductivity, high porosity and biomechanical compatibility [132]. For this requirement, autografts and allografts are used extensively for bone grafts. In the autograft technique, bone from another part will be harvested within the body, and this material fills the gap and provides optimal osteoinductivity, osteoconductivity and osteogenic properties. However, it has its own disadvantages: autografting often leads to complications in wound healing, additional surgery, donor pain and an inadequate supply of bone to fill the gap [133]. The main problem of the allograft technique has been linked with rejection problems and therefore use of immunogenic natural 572 polymer composite materials are becoming increasingly important as scaffolds 573 for bone tissue engineering. Next generation biomaterials should combine bioactive and bioresorbable materials, which mimic the natural function of bone and activate in vivo mechanisms of tissue regeneration. Composite materials based on combinations of biodegradable polymers and bioactive ceramics, including CTS and HAp, are discussed as suitable materials for scaffold fabrication. These composites exhibit tailored physical, biological and mechanical properties as well as predictable degradation behavior. The appropriate selection of a particular composite for a given application requires a detailed understanding of relevant cells and/or tissue response. An overview of these findings is 
presented and discussed in this review, highlighting the influence of material preparation methods, scaffold mechanical strength, in vitro activity of scaffold materials and chemical interaction with CTS polymer matrixes. The chapter also emphasizes future artificial bone materials, suggesting the utility of polymer composites in this field of biomaterials science. The limitations and concerns, such as adverse rejection reactions and the risk of acquiring transmissible diseases (AIDS and hepatitis) from tissues and fluids. have created substantial interest in the development of artificial materials as bone graft substitutes [134]. Very few compounds are classified as bioactive, biodegradable and osteoconductive. CTS and HAp are among the best bioactive biomaterials in bone tissue engineering and renowned for their excellent biocompatibility with the human body environment [135].

Various marine sources polysaccharides have been used for treatment of bone diseases like osteoporosis [136], arthritis [137], and so on. In order to create a moist environment for rapid wound healing, a hydrogel sheet composed of a blended powder of alginate, chitin/CTS and fucoidan has been developed as a functional wound dressing [138].

\subsubsection{Composite materials for bone tissue engineering}

Composite materials are now playing predominant role as scaffolds in bone tissue engineering. CTS has numerous advantageous properties for orthopedic applications, as described above and elsewhere [139], which make it ideal as a bone graft substituent. CTS scaffolds are flexible and their mechanical properties are inferior to those of normal bone, as it is unable to support load bearing bone implants. Moreover, CTS itself is not osteoconductive, although addition of ceramic materials improves its osteoconductivity and mechanical strength. CTS scaffolds alone cannot imitate all the properties of natural bone. The substantial development of composite materials with CTS mimics all the properties of bone. As proven, calcium phosphate materials are osteoconductive to mimic the inorganic portion of natural bone, while CTS / HAp composite materials show promise in mimicking the organic portion as well as the inorganic portion of natural bone (Figure 1). Several studies have been conducted with CTS/HAp composite materials for bone tissue engineering [140-145]. Calcium phosphate compounds are of great interest in the field of bone tissue engineering. $\mathrm{HAp}\left[\mathrm{Ca}_{10}\left(\mathrm{PO}_{4}\right)_{6}(\mathrm{OH})_{2}\right]$ is one of the most stable forms of calcium phosphate and it occurs in the bone as a major component (60-65 \%) [146]. HAp also possesses a variety of uses, including orthopedic, dental and maxillofacial applications. Therefore, HAp has recently emerged as an important compound for artificial bone preparation. It stimulates osteoconduction being gradually replaced by the host bone after implantation. It is being used for orthopedic replacements, especially in bone regeneration and dental implant treatment. The mechanical properties of HAp are poor, though, so it cannot be used for load bearing bone tissues. Polymers have been used to improve the mechanical properties of HAp (compressive 
strength, Young's modulus, fracture toughness) [147]. When CTS is combined with HAp, it might be able to mimic the function of natural bone.

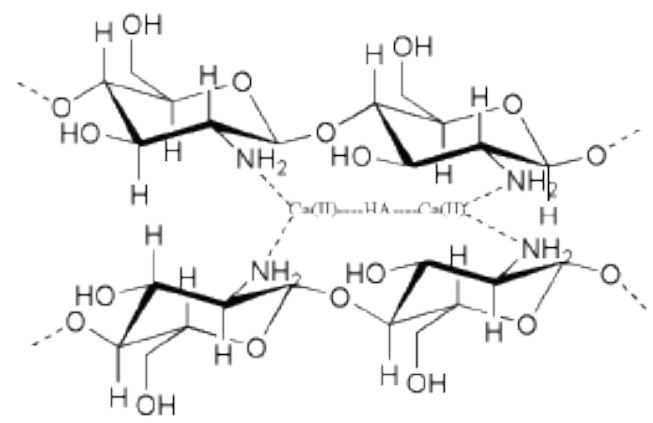

Figure 1. Chemical interaction between HAp and CTS

\subsubsection{Carbon nano tubes for bone tissue engineering}

Carbon nanotubes (CNTs) are allotropes of carbon with a cylindrical nanostructure and constructed with length-to-diameter ratio of up to $28,000,000: 1$. These cylindrical carbon molecules have novel properties, which make them potentially useful in many applications in nanotechnology, electronics, optics and materials sciences. CNTs have a high Young's modulus (1.0-1.8 TPa), high tensile strength (30-200 GPa) and high elongation at break (10-30\%). In addition, they have extremely small size (about 1-10 nm in diameter), high aspect ratio $(>1,000)$, high structural and chemical stability, and stiffness, as well as remarkable electrical, thermal, optical and bioactive properties $[148,149]$. All these properties make CNTs especially promising candidates as reinforcement fillers in the development of nano composites. It has been observed that the combination of CNT with CTS leads to an enormous increase in the mechanical strength of the composite [149].

CNTs hold great interest with respect to biomaterials, particularly those to be positioned in contact with bone such as prostheses for arthroplasty, plates or screws for fracture fixation, drug delivery systems, and scaffolding for bone regeneration. The most important concerns for the use of CNTs as a biomaterial are tissue safety, but only few reports have addressed the toxicity of CNTs. In particular, bone tissue compatibility is extremely important for using CNTs in biomaterials. Usui et al., who first developed CNT, found that these tubes have good bone tissue compatibility and are capable of permitting bone repair and becoming closely integrated with bone tissue and accelerate bone formation stimulated by recombinant human bone morphogenetic protein-2 [150]. 
A big challenge in the bone tissue engineering is mechanical strength improvement of the scaffold materials. One of the main purposes of creating CTS composites is to improve the mechanical strength of the material. CNT is a promising material to fulfill that gap due to its strong mechanical properties. Several authors have developed many composites as well as scaffold materials with CNT. It has been observed that cell adhesion on multi-walled CNT (MWCNT) coated dish is much higher than that on the collagen coated dish [151].

Our approach to the complexity of the developing suitable model for the in vitro TMJ prototype model to investigate excessive free radical generation and harnessing of the imbalance comes from a different angle: developing functional CTS based hydrogel system [84-94]. CTS is a natural cationic polysaccharide derived from chitin, with the structural composition being heavily influenced by the $\mathrm{pH}$ factors. The unique property of the CTS lies in its ability to form a controlled biofunctional interface with the dentin, enamel, restorative materials, as well as to the skin and other oral tissues. This process occurs through a favorable chemical and structural compatibility of the bioactive material (Figure 1). The biointeractive tissue influenced properties of CTS and its derivatives highlight an opportunity for development of dual functional restorative materials such as BIOF-INPs. The important points to address in developing the in vitro model to evaluate the amount of TMJ damaged caused by the oxidative stress as well as promote the repair of the fibrous cartilage and provide specific antimicrobial defense as well as pain management "in situ" and asses the cytotoxicity of the new materials lead us to design and investigate specific class of hydrogels and the preliminary results are summarized in the Figure 2. 

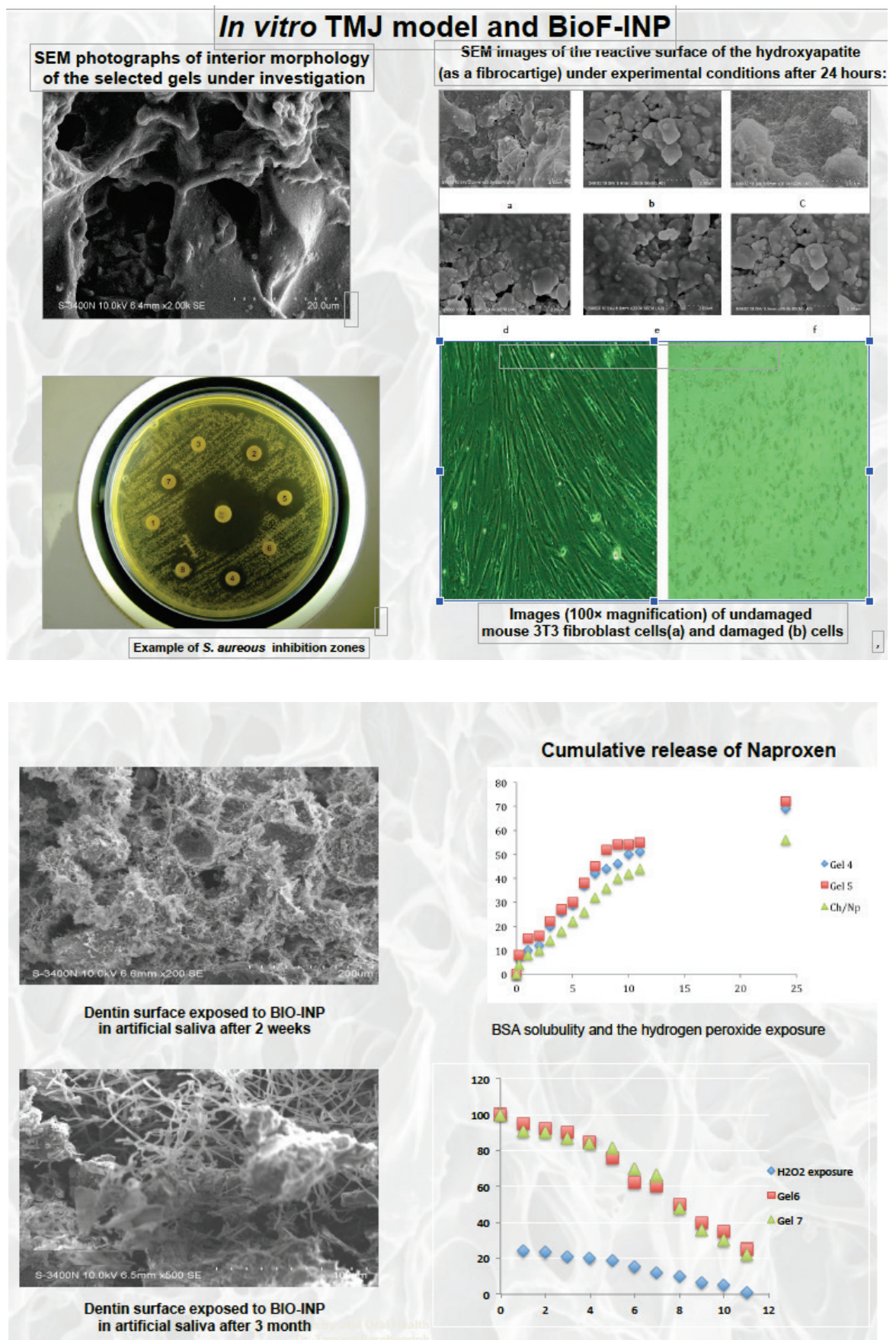

BSA solubulity and the hydrogen peroxide exposure

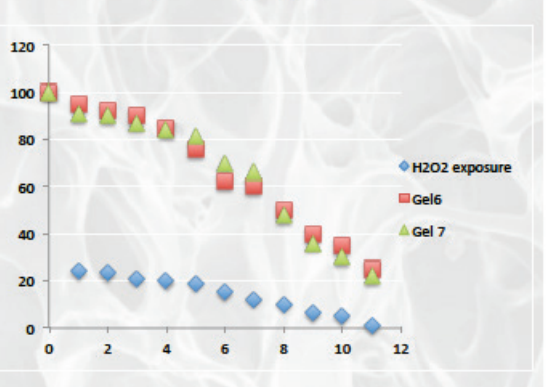

Figure 2. Summary of the performance of designer bioactive materials investigated in our group. a. SEM images of the prepared biomaterials. b. SEM images of the active hydroxyapatite surface exposed to artificial saliva. c. Microbiological evaluation of the prepared biomaterials. d. Cytotoxicity investigation summary of functionalized biomaterials. e. and f. Drug delivery capacity of the biomaterials evaluated in the study and evaluation of dentin regeneration capability of the materials in vitro. 
In summary the Figure 2 represents the scanning electron microscope (SEM) of the typical BIOF-INPs material designed and used in the study. The SEM images of the reactive surface of the dentin (as model of fibrous hard tissue) structure after application of the hydrogen (Figure $2 \mathrm{~b}$ immediately after application. Sample is kept in the artificial saliva for the entire time. The undamaged mouse 3T3 fibroblast cell(s) after application of the BIOF-INPs in the Figure 2a and damaged mouse 3T3 fibroblast cell(s) after application of the standard material in the Figure 2c. The example of Staphylococcus aureus inhibition zones and application of the therapeutic agent containing BIOF-INPs. Cumulative release of the Naproxen as a model therapeutic component of BIOF-IPN as summarized in the Figure 2e. The SEM images of the reactive surface of the dentin structure after application of the hydrogen (Figure $2 \mathrm{~d}$ immediately after application. Sample is kept in the artificial saliva for the entire time).

Present results demonstrate the capability of the BIOF-IPNs to play an important role in the functional multi-dimensional therapeutic restorative repair materials.

\subsection{CONCLUSION AND FUTURE DIRECTIONS}

This chapter brings together the advances in basic material science, biotechnology and designer functional materials with the specific aim of exploiting molecular mechanistic understanding of TMJ disorders with particular emphasis on the harnessing of excess of free radicals via functional biopolymers. The advantage of the intelligent designer functional materials represents amalgamation of molecular design, mechanism, bioanalytical advancement, molecular biology and ability to easily refocus the aim of the problem at hand make these materials only limited by imagination, creativity and multidimensional science linking scientist, health professionals and will only continue to progress when the recent advances will cross successfully the barrier from in vitro molecular design to in vivo application.

\section{REFERENCES}

1. M.C. Fletcher, J.F. Piecuch, S.E. Lieblich, in Peterson's Principles of Oral and Maxillofacial Surgery, $2^{\text {nd }}$ Ed., M.M. Hamilton (Ed.), BC Decker, Ontario, USA, 2004, pp. 933-947.

2. D.W. Howerton, M. Zysset. Oral Maxillofac. Surg. Clin. North Am. 1 (1989) 229-247.

3. D.M. Laskin. J. Oral Maxillofac. Surg. 56 (1998) 121.

4. $\quad$ D.M. Laskin. Oral Maxillofac. Surg. Clin. North Am. 7 (1995) 73-78.

5. $\quad$ S.F. Dworkin, L. LeResche. J. Craniomandib. Disord. 6 (1992) 301-355.

6. O.E. Ogle, M.B. Hertz. Oral Maxillofac. Surg. Clin. North Am. 12 (2000) 217-231. 
7. Fundamentals of Occlusion and Temporo-mandibular Disorders, J.P. Okeson (Ed.), C.V. Mosby, St. Louis, USA; 1985.

8. C.S. Stohler, in Oral and Maxillofacial Surgery. Temporomandibular Disorders, R.J. Fonseca et al. (Eds.), WB Saunders, Philadelphia, USA, 2000, pp. 38-45.

9. S.B. Milam, in Oral and Maxillofacial Surgery. Temporomandibular Disorders, R.J. Fonseca et al. (Eds.), WB Saunders, Philadelphia, USA, 2000, pp. 46-72.

10. D.M. Laskin. Oral Maxillofac. Surg. Clin. North Am. 5 (1994) 217-222.

11. P.L. Westesson, L. Eriksson, K. Kurita. Oral Surg. Oral Med. Oral Pathol. 68 (1989) 551-554.

12. C.H. Wilkes. Arch. Otolaryngol. Head Neck Surg. 115 (1989) 469-467.

13. C.S. Greene, D.M. Laskin. J. Am. Dent. Assoc. 117 (1988) 461-465.

14. S.B. Milam, J.P. Schmitz. J. Oral Maxillofac. Surg. 53 (1995) 1448-1454.

15. P.H. Chan. J. Cereb. Blood Flow Metab. 21 (2001) 2-14.

16. M.K. Irmak, E. Fadillioglu, M. Güleç. Cell Biochem. Funct. 20 (2002) 279-283.

17. M.M. Yaser, M.M. Randa, A. Belacy. J. Pharm. Biomed. Anal. 26 (2001) 605-608.

18. A. Pompella. Int. J. Vitam. Nutr. Res. 67 (1997) 289-297.

19. H. Sumii, H. Inoue, J. Onoue, A. Mori, T. Oda, T. Tsubokura. Hiroshima J. Med. Sci. 45 (1996) 51-55.

20. A.B. Holmlund, S. Axelsson. Int.J.Oral Maxillofac. Surg. 25(3) (1996) 266-271.

21. R. Emshoff. Oral Surg. Oral Med. Oral Pathol. Oral Radiol. Endod. 100(4) (2005) 409-414.

22. U. Voog, P. Alstergren, S. Eliasson, E. Leibur, R. Kallikorm, S. Kopp. Acta Odontol. Scand. 61(1) (2003) 57-64.

23. U. Voog, P. Alstergren, S. Eliasson, E. Leibur, R. Kallikorm, S. Kopp. Acta Odontol. Scand. 62(1) (2004) 7-13.

24. R.H. Tallents, D.J. Macher, S. Kyrkanides, R.W. Katzberg, M.E. Moss. J. Prosthet. Dent. 87(1) (2002).

25. S.J. Kim, Y.H. Park, S.P. Hong, B.O. Cho, J.W. Park, S.G. Kim. J. Oral Maxillofac. Surg. 61(10) (2003) 1156-1161.

26. D.I. Paegle, A.B. Holmlund, M.R. Östlund, L. Grillner. J. Oral Maxillofac. Surg. 62(4) (2004) 435-439.

27. E. Leibur, O. Jagur, P. Müürsepp, L. Veede, Ü. Voog-Oras. J. Cranio-Maxillofac. Surg. 38(8) (2010) 615-620.

28. P. Alstergren, S. Kopp, E. Theordosson. Acta Odontol. Scand. 57(1) (2003) 278-282.

29. A. Kamada, K. Kakudo, T. Arika, J. Okazaki, M. Kano, T. Sakaki. J. Cranio-MaxilloFac. Surg. 28(3) (2000) 247-248.

30. P. Alstergren, S. Kopp. Pain 72(1-2) (1997).

31. U. Voog, P. Alstergren, E. Leibur, R. Kallikorm, S. Kopp. Life Sci. 68(5) (2000) 591-602.

32. L.G.M. de Bont, G. Boering, R.S.B. Liem, F. Eulderink, P.-L. Westesson. J. Oral Maxillofac. Surg. 44 (1986) 634-643.

33. B. Stegenga, L.G.M. de Bont, G. Boering. J. Oral Maxillofac. Surg. 47 (1989) 249-256.

34. J.W. Nickerson, G. Boering. Oral Maxillofac. Surg. Clin. North Am. 1 (1989) 27-43.

35. B. Stegenga, L.G.M. de Bont, G. Boering, J.D. Van Willigen. J. Oral Maxillofac. Surg. 49 (1991) 1079-1088. 
36. H.A. Israel, C.J. Langevin, M.D. Singer, D.A. Behrman. J. Oral Maxillofac. Surg. 64 (2006) 1066-1074.

37. J.H. Quinn. Oral Maxillofac. Surg. Clin. North Am. 1 (1989) 47-57.

38. S. Kopp. J. Oral Maxillofac. Surg. 56 (1998) 189-191.

39. S.B. Milam. Oral Maxillofac. Surg. Clin. North Am. 12 (2000) 5-26.

40. J.J. Moses, D. Sartorius, R. Glass, T. Tanaka, I. Poker. J. Oral Maxillofac. Surg. 47 (1989) 674-678.

41. D.W. Nitzan, M.F. Dolwick, M.W. Heft. J. Oral Maxillofac. Surg. 48 (1990) 798-801.

42. J.P. McCain, B. Sanders, M.G. Koslin, J.D. Quinn, P.B. Peters, A. Thomas Indresano. J. Oral Maxillofac. Surg. 50 (1992) 926-930.

43. P.A. Brennan, V. Ilankovan. J. Oral Maxillofac. Surg. 64 (2006) 949-951.

44. S.B. Milam, G. Zardeneta, J.P. Schmitz. J. Oral Maxillofac. Surg. 56 (1998) 214-223.

45. T. Shibata, K.I. Murakami, E. Kubota, H. Maeda. J. Oral Maxillofac. Surg. 56 (1998) 209-213.

46. L.C. Dijkgraaf, G. Zardeneta, F.W. Cordewener, R.S.B. Liem, J.P. Schmitz, L.G.M. de Bont, S.B. Milam. J. Oral Maxillofac. Surg. 61 (2003) 101-111.

47. J.H. Quinn, in Modern Practice in Orthognathic and Reconstructive Surgery, W.H. Bell (Ed.), WB Saunders, Philadelphia, USA, 1992, pp. 471-481.

48. L.C. Dijkgraaf, L.G.M. de Bont, G. Boering, R.S.B. Liem. J. Oral Maxillofac. Surg. 53 (1995) 1182-1192.

49. L.C. Dijkgraaf, S.B. Milam, in Oral Maxillofacial Surgery Knowledge Update, vol 3., J.F. Piecuch (Ed.). Rosemont, IL: American Association of Oral and Maxillofacial Surgeons, 2001, pp. 5-28.

50. L.B. Kaban, C. Bouchard, M.J. Trellis. J. Oral Maxillofac. Surg. 67 (2009) 1966-1978.

51. D. Mehrotra, A.L. Chellappa, C. Gupta, D. Passi, S. Kumar. J. Oral Biol. Craniofac. Res. 2(3) (2012) 144-148.

52. V. Petrovic, P. Zivkovic, D. Petrovic, V. Stefanovic. Oral Surg. Oral Med. Oral Pathol. Oral Radiol. 114 (2012) e1-e9.

53. J. Glowacki, J.B. Mulliken. Plast. Reconstr. Surg. 92 (1993) 601.

54. M.I. Gray, A.M. Pizzanelli, A.J. Grodzinsky, R.C. Lee. J. Orthop. Res. 6 (1988) 777-792.

55. H.A. Israel. Oral Maxillofac. Surg. Clin. North Am. 1 (1989) 85-92.

56. W.Y. Yih. Oral Maxillofac. Surg. Clin. North Am. 1 (1989) 93-109.

57. H.K. Kleinman, D. Philp, M.P. Hoffman. Curr. Opin. Biotechnol. 14 (2003) 526-532.

58. B.B. Ward, S.E. Brown, P.H. Krebsbach. Oral Dis. 16 (2010) 709-716.

59. A.C. Jones, C.H. Arns, D.W. Hutmacher, B.K. Milthorpe, A.P. Sheppard, M.A. Knackstedt. Biomaterials. 30 (2009) 1440-1451.

60. J. Malda, T.B. Woodfield, F. van der Vloodt, F.K. Kooy, D.E. Martens, J.C. Tramper, C.A. van Blitterswijk, J. Riesle. Biomaterials 25 (2004) 5773-5780.

61. X. Li, Q. Feng, X. Liu, W. Dong, F. Cui. Biomaterials 27 (2006) 1917-1923.

62. D.M. Cruz, M. Gomes, R.L. Reis, D. Moratal, M. Salmerón-Sánchez, J.L. Gómez Ribelles, J.F. Mano. J. Biomed. Mater. Res. A 95 (2010) 1182-1193.

63. K. Tanaka, T. Goto, T. Miyazaki, Y. Morita, S. Kobayashi, T. Takahashi. J. Dent. Res. 90 (2011) 906e911. 
64. T. Fuji, T. Anada, Y. Honda, Y. Shiwaku, H. Koike, S. Kamakura, K. Sasaki, O. Suzuki. Tissue Eng. Part A. 15 (2009) 3525-3535.

65. J.R. Porter, T.T. Ruckh, K.C. Popat. Biotechnol. Prog. 25 (2009) 1539-1560.

66. H. Yoshimoto, Y.M. Shin, H. Terai, J.P. Vacanti. Biomaterials 24 (2003) 2077-2082.

67. L. Ciocca, D. Donati, M. Fantini, E. Landi, A. Piattelli, G. Iezzi, A. Tampieri, A. Spadari, N. Romagnoli, R. Scotti. J. Biomater. Appl. 28(2) (2012) 207-218.

68. K. Strebhardt, A. Ullrich. Nat. Rev. Cancer. 8(6) (2008) 473-480.

69. S.L. Kitson, D.J. Quinn, T.S. Moody, D. Speed, W. Watters, D. Rozzell. Chim. Oggi. 31(4) (2013) 30-36.

70. Y. Miao, M. Hylarides, D.R. Fisher, T. Shelton, H. Moore, D.W. Wester, A.R. Fritzberg, C.T. Winkelmann, T. Hoffman, T.P. Quinn. Clin. Cancer Res. 11(15) (2005) 5616-5621.

71. H.R. Maecke, J.C. Reubi. J. Nucl. Med. 52(6) (2011) 841-844.

72. R. Hooda, M. Tripathi, K. Kapoor. The Pharma Innovation 1(1) (2011) 14-21.

73. J.D. Smart. Adv. Drug Deliv. Rev. (1993) 11253-11270.

74. J. Hao, P.W. Heng. Drug Dev. Ind. Pharm. 29(8) (2003) 821-832.

75. F. Winau, O. Westphal, R. Winau. Microbes Infect. 6(8) (2004) 786-789.

76. V. Prajapati, M. Bansal, P. Sharma. Int. J. Pharmtech. Res. 4(2) (2012) 582-589.

77. R. Mohammad, in Biopolymers, M. Elnasahar (Ed.), Intech, Sciyo, Rijeka, Croatia, 2010, pp. 575-596.

78. V. Bansal, P. Sharma, N. Sharma, O. Pal, R. Malviya. Adv. Biol. Res. 5(1) (2011) 28-37.

79. J.H. Park, G. Saravanakumar, K. Kim, I.C. Kwon. Adv. Drug Deliv. Rev. 62(1) (2010) 28-41.

80. J. Balcerzak, M. Mucha. PCACD (2010) 15117-15125.

81. V.T. Perchyonok, S. Zhang, T.G. Oberholzer. Open Nanosci. J. (2013) 71-77.

82. V.T. Perchyonok, I. Lykakis, A. Postigo, Streamlining free radical green chemistry, The Royal Society of Chemistry, Cambridge, UK, 2012.

83. V.T. Perchyonok, S. Zhang, T.G. Oberholzer. Curr. Nanosci. 8(4) (2012) 541-547.

84. V.T. Perchyonok, S. Zhang, T.G. Oberholzer. Curr. Org. Chem. (2011) 162437-162439.

85. V.T. Perchyonok, S. Zhang, T.G. Oberholzer. Curr. Drug Deliv. 10(1) (2013) 144-150.

86. V.T. Perchyonok, V. Reher, S. Zhang, S.R. Grobler, T.G. Oberholzer, W. Massey. OJST 4(2) (2014) 73-83.

87. V.T. Perchyonok, S. Zhang, T.G. Oberholzer. Curr. Org. Chem. (2012) 162430-162436.

88. V.T. Perchyonok, S. Zhang, S.R. Grobler, T.G. Oberholzer, W. Massey. Eur. J. Gen. Dent. 3(1) (2014) 57-65.

89. V.T. Perchyonok, S. Zhang, N.J. Basson, S.R. Grobler, T.G. Oberholzer, W. Massey. Adv. Tech. Biol. Med. 2(1) (2014).

90. S.R. Grobler, A. Olivier, V.T. Perchyonok, D. Moodley. Int. J. Dent. Oral Sci. (2014) (In press).

91. V.T. Perchyonok, S. Zhang, N.J. Basson, S.R. Grobler, T.G. Oberholzer, W. Massey. OJST 4 (2014) 99-108.

92. V.T. Perchyonok, S. Zhang, S.R. Grobler, T.G. Oberholzer, W. Massey. Eur. J. Gen. Dent. 3(1) (2014) 57-65. 
93. V.T. Perchyonok, S. Zhang, S.R. Grobler, T.G. Oberholzer. Eur. J. Dent. 7(4) 2013 412-418.

94. C.K. Sen, G.M. Gordillo, S. Roy, R. Kirsner, L. Lambert, T.K. Hunt, F. Gottrup, G.C. Gurtner, M.T. Longaker. Wound Repair Regen. 17 (2009) 763-771.

95. M.N. Kumar, R.A. Muzzarelli, C. Muzzarelli, H. Sashiwa, A.J. Domb. Chem. Rev. 104 (2004) 6017-6084.

96. M.J.C. Fresno, A.D. Ramírez, M.M. Jiménez. Eur. J. Pharm. Biopharm. 54 (2002) 329-335.

97. V.M. Ndesendo, V. Pillay, Y.E. Choonara, R.A. Khan, L. Meyer, E. Buchmann, U. Rosin. Int. J. Pharm. 370 (2009) 151-159.

98. D.S. Jones, A.D. Woolfson, A.F. Brown, W.A. Coulter, C. McClelland, C.R. Irwin. J. Control. Release 67 (2000) 357-368.

99. H. Blanco-Fuente, S. Anguiano-Igea, F.J. Otero-Espinar, J. Blanco-Méndez. Int. J. Pharm. 142 (1996) 169-174.

100. X. Qi, R.F. Tester. J. Pharm. Pharm. Sci. 14 (2011) 60-66.

101. R.G. Riley, J.D. Smart, J. Tsibouklis, P.W. Dettmar, F. Hampson, J.A. Davis, G. Kelly, R.W. Wilber. Int J. Pharm. 217 (2001) 87-100.

102. K.R. Kamath, K. Park, in Encyclopedia of Pharmaceutical Technology, vol. 10, J. Warbrick, J.C. Boylan (Eds.), Marcel Dekker, New York, USA, 1994, pp. 133-163.

103. H.S. Ch'ng, K. Park, P. Kelly, J.R. Robinson. J. Pharm. Sci. 74 (1985) 399-404.

104. E.E. Hassan, J.M. Gallo. Pharm. Res. 7 (1990) 491-498.

105. A. Maltese, A. Borzacchiello, L. Mayol, C. Bucolo, F. Maugeri, L. Nicolais, L. Ambrosio. Biomaterials 27 (2006) 5134-5142.

106. M.J. Colthurst, R.L. Williams, P.S. Hiscott, I. Grierson. Biomaterials 21 (2000) 649-665.

107. J.J. Mao, W.V. Giannobile, J.A. Helms, S.J. Hollister, P.H. Krebsbach, M.T. Longaker, S. Shi. J. Dent. Res. 85(11) (2006) 966-979.

108. H. Abukawa, H. Terai, D. Hannouche, J.P. Vacanti, L.B. Kaban, M.J. Troulis. J. Oral Maxillofac. Surg. 61 (2003) 94-100.

109. Y. Weng, Y. Cao, C. Arevalo, M.P. Vacanti, C.A. Vacanti. J. Oral Maxillofac. Surg. 59 (2001) 185-190.

110. M.M. Bailey, L. Wand, C.J. Bode, K.E. Mitchell, M.S. Detamore. Tissue Eng. 13 (2007) 2003-2010.

111. S. Zhu, B. Zhang, C. Man, Y. Ma, J. Hu. Osteoarthr. Cartil. 19 (2011) 743-750.

112. H. Kang, Y.D. Bi, Z.Q. Li, M.Y. Qi, E.M. Peng. Chinese J. Stomatol. 46 (2011) 541-546.

113. W.C. Puelacher, J. Wisser, C.A. Vacanti, N.F. Ferraro, D. Jaramillo, J.P. Vacanti. J. Oral Maxillofac. Surg. 52 (1994) 1172.

114. N.M. Girdler. Scand. J. Rheumatol. 27 (1998) 446.

115. I.N. Springer, B. Fleiner, S. Jepsen, Y. Açil. Biomaterials 22 (2001) 2569.

116. A.J. Almarza, K.A. Athanasiou. Tissue Eng. 10 (2004) 1787-1795.

117. O. Gauthier, R. Müller, D.V. Stechow, B. Lamy, P. Weiss, J.M. Bouler, E. Aguado, G. Daculsi. Biomaterials 26(27) (2005) 5444-5453.

118. M. Mehta, K. Schmidt-Bleek, G.N. Duda, D.J. Mooney. Adv. Drug Deliv. Rev. 64 (2012) 1257-1276.

119. J. Venkatesan, Z.J. Qian, B. Ryu, K.N. Ashok, S.K. Kim. Carbohydr. Polym. 83(2) (2011) 569-577. 
120. N.A.M. Barakat, M.S. Khil, A.M. Omran, F.A. Sheikh, H.Y. Kim. J. Mater. Process. Technol. 209(7) (2009) 3408-3415.

121. K.S. Vecchio, X. Zhang, J.B. Massie, M. Wang, C.W. Kim. Acta Biomater. 3(6) (2007) 910-918.

122. X. Wang, J.S. Nyman, X. Dong, H. Leng, M. Reyes, Fundamental Biomechanics in Bone Tissue Engineering, K.A. Athanasiou (Ed.), Morgan \& Claypool, LaVergne, TN, 2010.

123. X. Cai, H. Tong, X. Shen, W. Chen, J. Yan, J. Hu. Acta Biomater. 5(7) (2009) 2693-2703.

124. N. Vachiraroj, J. Ratanavaraporn, S. Damrongsakkul, R. Pichyangkura, T. Banaprasert, S. Kanokpanont. Int. J. Biol. Macromol. 45(5) (2009) 470-477.

125. W.W. Thein-Han, R.D.K. Misra. Acta Biomater 5(4) (2009) 1182-1197.

126. Z. Li, H.R. Ramay, K.D. Hauch, D. Xiao, M. Zhang. Biomaterials 26(18) (2005) 3919-3928.

127. S. Panzavolta, M. Fini, A. Nicoletti, B. Bracci, K. Rubini, R. Giardino, A. Bigi. Acta Biomater. 5(2) (2009) 636-643.

128. H.W. Kim, H.E. Kim, V. Salih. Biomaterials 26(25) (2005) 52.

129. H.W. Kim, H.E. Kim, V. Salih. Biomaterials 26(25) (2005) 5221-5230.

130. K.S. Vecchio, X. Zhang, J.B. Massie, M. Wang, C.W. Kim. Acta Biomater. 3 (2007) 910-918.

131. N.A.M. Barakat, K.A., Khalil, F.A. Sheikh, A.M. Omran, B. Gaihre, S.M. Khil, H.Y. Kim. Mater. Sci. Eng. C 28 (2008) 1381-1387.

132. A. Di Martino, M. Sittinger, M.V. Risbud. Biomaterials 26 (2005) 5983-5990.

133. Q. Hu, B. Li, M. Wang, J. Shen. Biomaterials 25 (2004) 779-785.

134. S. Teng, E. Lee, B. Yoon, D. Shin, H. Kim, J. Oh. J. Biomed. Mater. Res. Part A 88 (2009) 569-580.

135. F. Chen, Z. Wang, C. Lin. Mater. Lett. 57 (2002) 858-861.

136. Y. Zhang, J.R. Venugopal, A. El-Turki, S. Ramakrishna, B. Su, C.T. Lim. Biomaterials 29 (2008) 4314-4322.

137. C. Xianmiao, L. Yubao, Z. Yi, Z. Li, L. Jidong, W. Huanan. Mater. Sci. Eng. C 29 (2009) 29-35.

138. L. Kong, Y. Gao, W. Cao, Y. Gong, N. Zhao, X. Zhang. J. Biomed. Mater. Res. Part A 75A (2005) 275-282.

139. I. Manjubala, I. Ponomarev, I. Wilke, K. Jandt. J. Biomed. Mater. Res. Part A Appl. Biomater. 8 (2008) 7-16.

140. W. Thein-Han, R. Misra. Acta Biomater. 5 (2009) 1182-1197.

141. J. Oliveira, M. Rodrigues, S. Silva, P. Malafaya, M. Gomes, C. Viegas, I. Dias, J. Azevedo, J. Mano, R. Reis. Biomaterials 27 (2006) 6123-6137.

142. S. Ding. J. Non-Cryst. Solids 353 (2007) 2367-2373.

143. I. Yamaguchi, K. Tokuchi, H. Fukuzaki, Y. Koyama, K. Takakuda, H. Monma, J. Tanaka. J. Biomed. Mater. Res. 55 (2001) 20-27.

144. S.-K. Kim, E. Mendis. Food Res. Int. 39 (2006) 383-393.

145. S. Nath, A. Dey, A.K. Mukhopadhyay, B. Basu. Mater. Sci. Eng. A (2009) 513-514, 197-201.

146. P. Ajayan, O. Zhou. Carbon Nanotubes 80 (2001) 391-425.

147. S. Samal, S. Bal. J. Miner. Mater. Charact. Eng. 7 (2008) 355-370.

148. S.F. Wang, L. Shen, W.D. Zhang, Y.J. Tong. Biomacromolecules 6 (2005) 3067-3072. 
149. Y. Usui, K. Aoki, N. Narita, N. Murakami, I. Nakamura, K. Nakamura, N. Ishigaki, H. Yamazaki, H. Horiuchi, H. Kato. Small 4 (2008) 240.

150. M. Terada, S. Abe, T. Akasaka, M. Uo, Y. Kitagawa, F. Watari. Dental Mater. J. 28 (2009) 82-88. 